
\title{
3 Research Square \\ CDC20 is a Potential Prognostic and Immunological Biomarker in Pan-Cancer
}

\section{Chen Tao}

First Affiliated Hospital of Nanchang University

\section{Hu Bing}

First Affiliated Hospital of Nanchang University

\section{Zhan Xiangpeng}

First Affiliated Hospital of Nanchang University

\section{Liu Xiaoqiang}

First Affiliated Hospital of Nanchang University

\section{Deng Wen}

First Affiliated Hospital of Nanchang University

\section{Long Jiahui}

First Affiliated Hospital of Nanchang University

\section{Jiang Ming}

First Affiliated Hospital of Nanchang University

\section{Xiong Yunqiang}

First Affiliated Hospital of Nanchang University

\section{Chen Luyao}

First Affiliated Hospital of Nanchang University

Bin Fu ( $\nabla$ urofbin@163.com )

First Affiliated Hospital of Nanchang University

\section{Research}

Keywords: CDC20, pan-cancer, TMB, MSI, tumor microenvironment.

Posted Date: September 30th, 2021

DOI: https://doi.org/10.21203/rs.3.rs-917402/v1

License: (9) This work is licensed under a Creative Commons Attribution 4.0 International License. Read Full License 


\section{Abstract}

\section{Background}

CDC20(cell division cycle 20 homologue) plays a vital role in the cell cycle progression through targeting key substrates for destruction. Current studies have shown that CDC20 functions as an oncogene in various cancers. However, the potential correlations of $\mathrm{CDC} 20$ with prognosis and immune infiltrates in different cancers remain unclear.

\section{Result}

CDC20 expression was higher in most cancers, compared with normal tissues, and the high expression of CDC20 was correlated with poor prognosis and a higher pathological stage. Furthermore, there were significant correlations between $\mathrm{CDC} 20$ dysregulation with tumor mutation burden(TMB), microsatellite instability(MSI), tumor microenvironment and tumor- and immune-related genes.

\section{Conclusion}

CDC20 may be used as a potential prognostic and immunotherapeuticbiomarker which affects tumor progression.

\section{Introduction}

Cancer has gradually become the leading cause of morbidity and mortality worldwide, which has a tremendous impact on our health and economy [1]. Traditional first-line therapies include surgery, chemotherapy and radiotherapy for most cancer patients. At present, in the wake of developments in medical technology and the emergence of immune checkpoint inhibitors(ICls), cancer immunotherapy has become more and more important [2]. Immune checkpoint therapy is a kind of treatment method to improve the anti-tumor immune response by regulating the activity of $T$ cells. Currently, cytotoxic $T$ lymphocyte associated antigen 4 (CTLA-4) and programmed cell death protein 1 (PD-1) are the most concerned immune checkpoints in the treatment of immune checkpoint therapy. In the drug application of immune checkpoint therapy, there are mainly CTLA-4 monoclonal antibody ipilimumab for the treatment of patients with malignant melanoma [3], PD-1 blocking monoclonal antibody pembrolizumab for nonsmall cell lung cancer expressing PD-L1 [4], nivolumab targeting PD-1 for the treatment of patients with malignant kidney cancer [5], and atezolizumab targeting PD-L1 for the treatment of patients with advanced or deteriorating disease [6]. For some cancer patients who do not respond to conventional chemotherapy, these drugs can often achieve certain efficacy. Therefore, it is urgent to find more effective therapeutic targets for patient prognosis and treatment.

CDC20 includes seven WD40 repeats, which are necessary to mediate protein-protein interactions [7]. In order to exert its biological functions, CDC20 targets its downstream substates for ubiquitination and subsequent degradation, which plays an indispensable role in regulation of cell cycle, apoptosis, brain 
development and DNA damage repair [8-11]. The present studies reported that CDC20 exhibited its oncogenic function in most human tumorigenesis $[12,13]$. And its overexpression was associated with invasiveness in pancreatic cancer [14], breast cancer [15], prostate cancer [16], lung cancer [17] and so on. However, to date, there is no systematical and comprehensive study on the potential function of CDC20 in pan-cancer.

In this study, we comprehensively analyzed the expression level of CDC20 and investigated the correlations of CDC20 expression with clinical prognosis level, tumor mutation, immune microenvironment, and various tumor- and immune-related genes. Ultimately, by Gene Set Enrichment Analysis (GSEA), the biological function and pathways of ZIC2 were explored.

\section{Results}

\section{CDC20 mRNA levels in pan-cancers}

Firstly, Oncomine database was applied to explore CDC20 gene expression in pan-cancer. Our results revealed that CDC20 expression was higher in bladder cancer, brain and CNS cancer, breast cancer, cervical cancer, colorectal cancer, esophageal cancer, gastric cancer, head and neck cancer, liver cancer, lung cancer, lymphoma, ovarian cancer, pancreatic cancer, prostate cancer and sarcoma. Only in leukemia and myeloma, CDC20 expression was lower compared to the corresponding normal tissues (Figure 1A). In order to further analyze CDC20 expression in pan-cancer, the mRNA levels of CDC20 in 33 cancer samples from TCGA database were also obtained. Our results indicated that the expression of CDC20 in 19 tumors (BLCA, BRCA, CESC, CHOL, COAD, ESCA, GBM, HNSC, KIRC, KIRP, LIHC, LUAD, LUSC, PRAD, READ, SARC, STAD, THCA, UCEC) is higher in cancer versus adjacent normal tissues. And there was no significant difference between the expression of CDC20 and five cancers (KICH, PAAD, PCPG, SKCM and THYM) (Figure 1B). Due to the lack of normal tissue control in adrenocortical carcinoma (ACC), lymphoid neoplasm diffuse large B-cell lymphoma (DLBC), acute myeloid leukemia (LAML), brain lower grade glioma (LGG), mesothelioma (MESO), ovarian serous cystadenocarcinoma (OV), testicular germ cell tumors (TGCT), uterine carcinosarcoma (UCS), and uveal melanoma (UVM), we used the GEPIA2 to explore the expression difference between these tumor tissues and the corresponding normal tissues of the GTEx database. We found that CDC20 expression was also higher in ACC, DLBC, LAML, LGG, OV, PAAD, SKCM, THYM, UCS. (Figure 1C). In addition, cBioPortal database was utilized to further analyze the changes on CDC20 expression. As shown in Figure 1D, the highest alteration ratio was associated with amplification, followed by mutation and deep deletion and the highest alteration frequency was showed in ovarian cancer cases.

\section{Multifaceted prognostic value of CDC20 in pan-cancers}

In order to explore the correlation between the CDC20 expression and the prognosis of patients including OS, DSS, DFI, and PFI, the Kaplan-Meier (KM) survival curve and Cox proportional hazards models were utilized. The univariate cox regression analysis indicated that the upregulated $\mathrm{CDC} 20$ expression was negatively correlated with OS in ACC $(P<0.001), \mathrm{KICH}(P<0.001), \mathrm{KIRC}(P<0.001), \mathrm{KIRP}(P<0.001)$, LGG 
( $P<0.001)$, LIHC $(P<0.001)$, LUAD ( $P=0.001)$, MESO $(P<0.001)$, PAAD $(P<0.001)$, SARC $(P=0.012)$, SKCM $(P=0.009)$, UCEC $(P=0.049)$ and positively with OS in THYM $(P=0.03)$ (Figure $2 A)$, negatively with $D S S$ in ACC $(P<0.001), \operatorname{KICH}(P<0.001), \operatorname{KIRC}(P<0.001), \operatorname{KIRP}(P<0.001), \operatorname{LGG}(P<0.001)$, LIHC $(P<0.001)$, LUAD $(P=0.001)$, MESO $(P<0.001)$, PAAD $(P<0.001)$, PCPG $(P=0.023)$, PRAD $(P=0.031)$, SARC $(P=0.019)$, SKCM $(P=0.016)$, THCA $(P=0.049)$ (Figure $3 A)$, negatively with $D F I$ in ACC $(P=0.019), B R C A(P=0.016), K I R P$ $(P<0.001)$, LIHC $(P<0.001)$, PAAD $(P=0.02)$, PRAD $(P<0.001)$, SARC $(P=0.004)$, THCA $(P<0.001)$, UCEC $(P=0.043)$ (Figure $4 A)$, and negatively with $P F I$ in ACC $(P<0.001), B R C A(P=0.030), K I C H(P<0.001), K I R C$ $(P<0.001)$, KIRP $(P<0.001)$, LGG $(P<0.001)$, LIHC $(P<0.001)$, LUAD $(P=0.043)$, MESO $(P<0.001), P A A D$ $(P<0.001), P C P G(P=0.002)$, PRAD $(P<0.001)$, SARC $(P=0.001)$, THCA $(P<0.001)$ and UCEC $(P=0.002)$

(Figure 5A). K-M survival analysis suggested that high expression of $\mathrm{CDC} 20$ was associated with poor OS time in ACC, KIRC, KIRP, LGG, LIHC, LUAD, MESO and SKCM and low expression of CDC20 was related to poor OS time only in DLBC and THYM (Figure 2B-2K). We also estimated the relationship between CDC20 expression and DSS, the K-M curve showed that the increased $C D C 20$ expression was related with poor DSS in 7 types of cancer (ACC, KIRC, KIRP, LGG, LIHC, LUAD and MESO) and only showed that the low expression of CDC20 was associated with poor DSS time in DLBC (Figure 3B-3I). In addition, in the analysis of the relationship between DFI and CDC20 expression, the K-M survival analysis revealed that the high CDC20 expression corresponded with poor PFI in ACC, KIRP, LIHC, MESO, PRAD, SARC and THCA (Figure 4B-4H). Finally, we assessed the PFI in 33 tumors,the results from K-M analysis showed that higher levels of CDC20 mRNA was related with worse PFI in ACC, KIRC, KIRP, LGG, LIHC, MESO, PAAD, PRAD, SARC, THCA and UCEC (Figure 5B-5L).

\section{Correlations between CDC20 expression and clinicopathology}

To explore the relationship of CDC20 expression and clinicopathologic stages in diverse cancers, we obtained the CDC20 expression in stage I, II, III, and IV. As shown in Figure 6, there were significant differences in CDC20 expression between stage I and II in BRCA, LIHC, LUAD, PAAD, READ, SKCM and TGCT, between stage I and III in BRCA, KIRC, KIRP, LIHC, LUAD, SKCM and TGCT, between stage I and IV in ACC, KICH, KIRC and KIRP, between stages II and III in ACC, KIRC, KIRP and ESCA, between stages II and IV inACC, KICH, KIRC, KIRP and LIHC, between stages III and IV in KICH, KIRC and LIHC. Notably, CDC20 expression upregulated along with the increase of tumor grade in $\mathrm{ACC}, \mathrm{BRCA}, \mathrm{KICH}, \mathrm{KIRC}, \mathrm{KIRP}, \mathrm{LIHC}$, LUAD and TGCT.

\section{Association of CDC20 expression with TMB, MSI.}

TMB and MSI are closely associated with response to immunotherapy, which affect tumor prognosis[19, 20].Therefore, we calculated the TMB and MSI of each sample and investigated the relationship between CDC20 expression and TMB and MSI in pan-cancer. The results showed that the high CDC20 expression was positively correlated with theTMB in ACC, BLCA, BRCA, CESC, COAD, KICH, KIRC, LAML, LGG, LIHC, LUAD, LUSC, PAAD, PRAD, SARC, SKCM, STAD, TGCT, UCEC and UCS, while only negatively correlated with the TMB in THYM (Figure 7A).Moreover, CDC20 expression had a positive correlation with the MSI in 
BLCA, BRCA, COAD, HNSC, LIHC, SARC, STAD and UCEC, while only had a negative correlation with the $M S I$ in READ (Figure 7B).

\section{Correlation of CDC20 expression and tumor microenvironment (TME) and immune cell infiltration}

The abnormal alterations of TME play a vital role in cancer cell progression and metastasis[21]. To analyze the relationship between TME and CDC20 expression in pan-cancer, the ESTIMATE algorithm was applied to calculate stromal and immune cell scores. As shown in Figure 8A, CDC20 expression positively correlated with stromal score and immune score in LGG and THCA, and negatively in GBM, LUSC and STAD. However, in BRCA and THYM, the expression of CDC20 negatively correlated with stromal score and positively correlated with immune score. The detailed resultsare shown in Supplementary Table S1.In addition, we acquired the content of 22 specific immune cells to further explore the association between $\mathrm{CDC} 20$ expression and immune cell infiltration in pan-cancer. As shown in Figure 8B, the four tumors (BRCA, KIRC, THYM and LUAD) with the strongest correlation were displayed. In other cancers, the relationship of CDC20 expression and immune cell infiltrating is shown in Supplementary Table S2. Our results suggested that the expression of CDC20 was closely relevant to immune infiltration levels.

\section{Correlations of $\mathrm{CDC} 20$ expression with some specific genes}

In order to explore the relationship of CDC20 expression and immune checkpoint genes, we conducted gene co-expression analyses. Our result suggested that CDC20 expressionwas significantly associated with immune-related factors in most cancers (Figure 9A) (Supplementary Table S3). As an intracellular mismatch repair mechanism and epigenetic modification respectively, MMRs and DNA methylation play an important role in tumor genesis. We explored the correlations of CDC20 expression with DNA methylation and MMRs by completing the co-expression analysis of CDC20 with 5 MMRs genes (MLH1, MSH2, MSH6, PMS2 and EPCAM) and 5 methyltransferases (DNMT1, TRDMT1, DNMT3A, DNMT3B and DNMT3L). Our results indicated that the expression of CDC20 was relevant to the expression of them (Figure 9B-9C) (Supplementary Table S4-S5). m6A is a base modification behavior widely existing in mRNA and the internal modification of mRNA is used to maintain the stability of mRNA, which affects tumor development. Our results showed that the expression of CDC20 was significantly correlated with the mRNA m6A related genes (Figure 9D) (Supplementary Table S6).

\section{GSEA analysis}

To further explore the functional and pathway enrichment analyses of CDC20, GSEA was used to perform KEGG enrichment analyses. As shown in Figure 10, immune-related pathways were differently enriched in cytokine receptor interaction, Tcellreceptorsignalingpathway, antigen processing and presentation,JAKSTAT signaling pathway, regulation of autophagy, chemokine signaling pathway, RIG I like receptor signaling pathway.In addition to immune-related pathways, CDC20 also affected other pathways,includingolfactorytransduction, cell cycle, ascorbateandaldaratemetabolism, pentoseandglucuronateinterconversions and mismatchrepair. 


\section{Discussion}

Ubiquitination is considered to a key role in regulating a variety of cellular processes, including cell cycle progression, apoptosis, cell proliferation, DNA damage, migration as well as invasion [22, 23]. As the most complicated E3 ubiquitin ligase, the Anaphase Promoting Complex (APC) has been supposed to be the chief driving force regulating the process of cell cycle $[24,25]$. In order to exert its biological function, APC core is related with two activators ( $\mathrm{CDC} 20$ and $\mathrm{CDH} 1)$, resulting in two different $\mathrm{E} 3$ ubiquitin ligase complexes (APC ${ }^{\mathrm{CDC} 20}$ and $\mathrm{APC}{ }^{\mathrm{CDH} 1}$ ), respectively [13]. $\mathrm{CDC20}$ targets its downstream substrates for ubiquitination and subsequent degradation to performs various biological functions, which include regulation of cell cycle, apoptosis, DNA damage repair and invasion [26]. For instance, the transition from metaphase to anaphase was regulated by CDC20 through targeting Securin and Cyclin B for ubiquitination and degradation [27] and $\mathrm{Cdc} 20$ targeted $\mathrm{Mcl}-1$ and $\mathrm{BIM}$ to participate in the regulation of apoptosis $[9,28]$. In addition, up-regulation of CDC20 was found to be associated with poor prognosis in various types of cancers [29-31]. Considering that previous studies rarely involved the prognostic value, biological function and association with immune microenvironment of CDC20 in pan cancer, it is crucial to explore the potential mechanism of its dysregulation.

In our current study, we first found that CDC20 was highly expressed in up to 28 tumors compared with normal tissues, indicating CDC20 plays an oncogenic role in pan-cancers. Second, we explored the relationship between the expression of $C D C 20$ and OS, DSS, DFI and PFI. Our results showed that high expression of $\mathrm{CDC} 20$ was associated with poor prognosis in most tumors. Third, we further revealed that in ACC, BRCA, KICH, KIRC, KIRP, LUAD and TGCT, the expression of CDC20 increased with the increase of pathological stage. Fourth, TMB can indirectly reflect the tumor's ability to produce new antigens and predict the efficacy of immunotherapy for a variety of tumors [32]. Tumor patients with high TMB have a higher clinical benefit rate after receiving immune checkpoint inhibitor treatment [33, 34]. Similarly, MSI is also an important biological marker to predict the efficacy of immunotherapy [35, 36]. Our study suggested that CDC20 expression is associated with TMB in 21 cancers and with MSI in 9 cancers. Fifth, More and more evidence show that tumor microenvironment (TME) plays a key role in tumor progression, such as local drug resistance, immune escape and cancer metastasis [37]. We analyzed the correlation between CDC20 expression and TME and immune cells' infiltration, finding that the expression of CDC20 affects the activity of stromal cells and immune cells in most cancers. Sixth, our study also revealed the CDC20 expression is co-expressed with immune checkpoint related genes, MMRs gene, DNA methyltransferase gene and M6A RNA methylation related genes, which are closely related to immunotherapy. All in all, our results above suggested that CDC20 may provide an effective novel target for immunotherapy. Finally, few studies have reported the relationship between CDC20 expression and immune microenvironment. By using GSEA, we found that the pathogenic mechanism of CDC20 was enriched in immune related pathways including cytokine receptor interaction, $T$ cell receptor signaling pathway, antigen processing and presentation, JAK-STAT signaling pathway, regulation of autophagy, chemokine signaling pathway, RIG I like receptor signaling pathway. 
At present, there are many clinical and basic studies which are consistent with our summary results. In pancreatic cancer, over expression of CDC20 was detected compared with normal tissues, and CDC20 depletion inhibited cell growth and induced G2/M cell cycle arrest [14,38]. Similarly, CDC20 was highly expressed in breast cancer cells and its overexpression was correlated with invasive processes [30]. In addition, CDC20 has been identified to be associated with prostate tumorigenesis. Studies have shown that through the interaction between APC inhibitor Daxx and CDC20, the degradation of APC CDC20 substrates was inhibited, which led to the transient delay of mitotic process and chromosome instability. Consistently, High expression of Daxx was shown in prostate cancer tissues and positively associated with the Gleason score and metastasis [39]. Meanwhile, Zhang et al also reported that the expression of CD44 + prostate cancer stem cells was inhibited by reducing CDC20 expression [40]. CDC20 was also highly expressed in primary cutaneous squamous cell carcinoma, promoting the migration and invasion of tumor cells by the Wnt/ $\beta$-catenin signaling pathway [41]. In diffuse large B-cell lymphoma, CDC20, as a key downstream gene of MDM2-p53 signaling pathway, regulated tumor cell proliferation, apoptosis and cell cycle changes [42]. One study has found that in hepatocellular carcinoma, the increased expression of $\mathrm{CDC} 20$ affected tumor progression through regulating epithelial-mesenchymal transition [43]. Another study displayed that the consumption of endogenous CDC20 decreased the cell proliferation of hepatocellular carcinoma cells and induced the G2 / M cell cycle stage [44]. As the most common cause of cancer death, lung cancer can be divided into non-small cell lung cancer (NSCLC) and small cell lung cancer (SCLC). Studies have shown that CDC20 was highly expressed and may be a potential prognostic marker of human NSCLC [31]. In addition, Kidokoro et al found that by knocking down CDC20, cell growth was inhibited, G2 / M cell cycle arrest was induced and the colony formation of lung cancer cells was delayed [17]. High expression of CDC20 was also founded in bladder cancer cells, targeting FoxO1 degradation to induce radioresistance [45]. All the above studies can be used as the external validation of our research, showing that $\mathrm{CDC} 20$ exert its oncogenic function in various tumors. However, there are certain limitations in our research. Firstly, our research is completely based on public database, and we need further experiments to verify it. Secondly, the evaluation of CDC20 expression is based on mRNA level, which is difficult to reflect the level of functional protein.

\section{Conclusion}

In summary, our analysis shows that CDC20 is highly expressed in a variety of tumors, and the high expression of CDC20 is closely related to poor prognosis, pathological stage, TMB, MSI, immune cell infiltration, immune checkpoint genes and immune related pathways. This study can provide a new insight for future immunotherapeutic design.

\section{Materials And Methods}

\section{CDC20 expression in pan-cancer}

In order to detect CDC20 differential expression in different cancers, we initially applied the Oncomine database (http://www.oncomine.org). From the UCSC Xena project (http://xena.ucsc.edu), we acquired 
the transcriptome data, mutation data and clinicopathological data of 33 kinds of cancer in TCGA for further study. By using Perl software, we extracted and integrated CDC20 expression levels and used the R-package "ggpubr" to visualize in the form of box diagram. For some certain tumors without normal or with highly limited normal tissues, the "Expression analysis-Box plots" module of the GEPIA2 web server (http://gepia2.cancer-pku.cn) was applied, where we acquired box plots of the expression difference between the these tumor tissues and the corresponding normal tissues of the GTEx database. According the cBioPortal database (https://www.cbioportal.org), we analyzed the genetic alteration characteristics of $\mathrm{CDC} 20$ further.

\section{Prognostic and Clinicopathological correlation Analyses}

To explore the correlations between CDC20 expression and patients' overall survival (OS), disease-specific survival (DSS), disease-free interval (DFI) and progression-free interval (PFI) in 33 types of cancer, forest plots and Kaplan-Meier curves were utilized. By using univariate survival analysis, we computed the hazard ratios (HRs) and 95\% confidence intervals.In addition, for further clinical correlation analysis, we obtained the clinical correlation data from TCGA database to explore the clinical correlation between CDC20 expression level and tumor stage using the R-package "limma" and "ggpubr".

\section{TMB and MSI value calculation}

To perform TMB and MSI analysis, we downloaded the somatic mutation data and the MSI score of 33 cancers from TCGA database and used the Perl language and the R-package " $\mathrm{fmsb}$ " to calculate the total tumor mutational burden. A correlation analysis between CDC20 expression level and TMB or MSI was visualized by a radar map.

\section{Evaluation of immune microenvironment and immune cells infiltration}

The ESTIMATE algorithm in R-package "estimate" and "limma" was applied to count immune and stromal cell scores for each tumor sample [18]. By using CIBERSORT, we performed the estimation of immune cell infiltration in pan-cancer. The R-package "ggplot2", "ggpubr" and "ggExtra" were utilized to perform the correlation analysis of CDC20 expression with the tumor immune microenvironment and immune cell infiltration.

\section{Gene Set Enrichment Analysis (GSEA) of CDC20 in pan-cancer}

We obtained the GO gene and KEGG gene sets from the GSEA database (https://www.gsea-msigdb.org/). By using R-package "limma", "org.Hs.eg.db", "clusterprofiler" and "enrichplot", we conducted GO and KEGG functional annotation.

\section{Statistical methods}

The Perl language and the $\mathrm{R}$ language (Version 3.6.3) were utilized for data processing, analysis and visualization. We performed the gene expression analysis of CDC20 by the Wilcox test. And we conducted 
the correlation analysis by pearson correlation coefficient test. $P \leq 0.05$ was considered statistically significant.

\section{Abbreviations}

ACC Adrenocortical Carcinoma;

BLCA Bladder Urothelial Carcinoma;

BRCA Breast invasive carcinoma;

CESC Cervical squamous cell carcinoma and endocervical adenocarcinoma;

CHOL Cholangiocarcinoma;

COAD Colon adenocarcinoma;

DFI Disease-Free Interval;

DLBC Lymphoid Neoplasm Diffuse Large B-cell Lymphoma;

DSS Disease-Specific Survival;

ESCA Esophageal carcinoma;

GBM Glioblastoma multiforme;

GEO Gene Expression Omnibus;

GSEA Gene set enrichment analysis;

HNSC Head and Neck squamous cell carcinoma;

KICH Kidney Chromophobe;

KIRC Kidney renal clear cell carcinoma;

KIRP Kidney renal papillary cell carcinoma;

K-M Kaplan-Meier curves;

LAML Acute Myeloid Leukemia;

LGG Brain Lower Grade Glioma;

LIHC Liver hepatocellular carcinoma;
LUAD Lung adenocarcinoma;

LUSC Lung squamous cell carcinoma;

MESO Mesothelioma;

MMRs Mismatch repair system;

MSI Microsatellite instability;

OS Overall Survival;

OV Ovarian serous

cystadenocarcinoma

PAAD Pancreatic adenocarcinoma;

PCPG Pheochromocytoma and Paraganglioma

PFI Progression-Free Interval;

PRAD Prostate adenocarcinoma;

READ Rectum adenocarcinoma;

SARC Sarcoma;

SKCM Skin Cutaneous Melanoma;

STAD Stomach adenocarcinoma;

TMB Tumor mutation burden;

TCGA The Cancer Genome Atlas;

TGCT Testicular Germ Cell Tumors;

THCA Thyroid carcinoma;

THYM Thymoma;

UCEC Uterine Corpus Endometrial Carcinoma;

UCS Uterine Carcinosarcoma;

\section{Declarations}


Acknowledgements

Not applicable

\section{Authors' contributions}

CT and HB conceived the project; ZXP and HB explored the data; CT, JM and LJH carried out the bioinformatics analysis; LXQ, DW and XYQ provide technical support; FB and CLY revised the manuscript and as corresponding author. All authors read and approved the final manuscript.

\section{Funding}

This work was supported by the National Natural Science Foundation of China (81960512).

\section{Data availability}

Our analyses are based on public online databases. All the data in this study are available from UCSC Xena (https://xena.ucsc.edu/) and Kaplan-Meier Plotter (https://kmplot.com/analysis/)

\section{Ethics approval and consent to participate}

Not applicable

\section{Consent to participate}

Not applicable

\section{Conflict of interest}

The author declares that there is no conflict of interest with any financial organization or corporation or individual that can inappropriately influence this work

\section{References}

1. Sung H, Ferlay J, Siegel RL, Laversanne M, Soerjomataram I, Jemal A, Bray F: Global Cancer Statistics 2020: GLOBOCAN Estimates of Incidence and Mortality Worldwide for 36 Cancers in 185 Countries. CA: a cancer journal for clinicians 2021, 71(3):209-249.

2. Pardoll DM: The blockade of immune checkpoints in cancer immunotherapy. Nature reviews Cancer 2012, 12(4):252-264.

3. Tawbi HA, Forsyth PA, Algazi A, Hamid O, Hodi FS, Moschos SJ, Khushalani NI, Lewis K, Lao CD, Postow MA et al: Combined Nivolumab and Ipilimumab in Melanoma Metastatic to the Brain. The New England journal of medicine 2018, 379(8):722-730.

4. Gandhi L, Rodríguez-Abreu D, Gadgeel S, Esteban E, Felip E, De Angelis F, Domine M, Clingan P, Hochmair MJ, Powell SF et al: Pembrolizumab plus Chemotherapy in Metastatic Non-Small-Cell 
Lung Cancer. The New England journal of medicine 2018, 378(22):2078-2092.

5. Motzer RJ, Escudier B, McDermott DF, George S, Hammers HJ, Srinivas S, Tykodi SS, Sosman JA, Procopio G, Plimack ER et al: Nivolumab versus Everolimus in Advanced Renal-Cell Carcinoma. The New England journal of medicine 2015, 373(19):1803-1813.

6. Balar AV, Galsky MD, Rosenberg JE, Powles T, Petrylak DP, Bellmunt J, Loriot Y, Necchi A, HoffmanCensits J, Perez-Gracia JL et al: Atezolizumab as first-line treatment in cisplatin-ineligible patients with locally advanced and metastatic urothelial carcinoma: a single-arm, multicentre, phase 2 trial. Lancet (London, England) 2017, 389(10064):67-76.

7. Hartwell LH, Mortimer RK, Culotti J, Culotti M: Genetic Control of the Cell Division Cycle in Yeast: V. Genetic Analysis of cdc Mutants. Genetics 1973, 74(2):267-286.

8. Nasmyth K: Disseminating the genome: joining, resolving, and separating sister chromatids during mitosis and meiosis. Annual review of genetics 2001, 35:673-745.

9. Wan L, Tan M, Yang J, Inuzuka H, Dai X, Wu T, Liu J, Shaik S, Chen G, Deng J et al: APC(Cdc20) suppresses apoptosis through targeting Bim for ubiquitination and destruction. Developmental cell 2014, 29(4):377-391.

10. Yang Y, Kim AH, Yamada T, Wu B, Bilimoria PM, Ikeuchi Y, de la Iglesia N, Shen J, Bonni A: A Cdc20APC ubiquitin signaling pathway regulates presynaptic differentiation. Science (New York, NY) 2009, 326(5952):575-578.

11. Chun AC, Kok KH, Jin DY: REV7 is required for anaphase-promoting complex-dependent ubiquitination and degradation of translesion DNA polymerase REV1. Cell cycle (Georgetown, Tex) 2013, 12(2):365-378.

12. Penas C, Ramachandran V, Ayad NG: The APC/C Ubiquitin Ligase: From Cell Biology to Tumorigenesis. Frontiers in oncology 2011, 1:60.

13. Wang Z, Wan L, Zhong J, Inuzuka H, Liu P, Sarkar FH, Wei W: Cdc20: a potential novel therapeutic target for cancer treatment. Current pharmaceutical design 2013, 19(18):3210-3214.

14. Taniguchi K, Momiyama N, Ueda M, Matsuyama R, Mori R, Fujii Y, Ichikawa Y, Endo I, Togo S, Shimada H: Targeting of CDC2O via small interfering RNA causes enhancement of the cytotoxicity of chemoradiation. Anticancer research 2008, 28(3a):1559-1563.

15. Yuan B, Xu Y, Woo JH, Wang Y, Bae YK, Yoon DS, Wersto RP, Tully E, Wilsbach K, Gabrielson E: Increased expression of mitotic checkpoint genes in breast cancer cells with chromosomal instability. Clinical cancer research : an official journal of the American Association for Cancer Research 2006, 12(2):405-410.

16. Wang Q, Tiffen J, Bailey CG, Lehman ML, Ritchie W, Fazli L, Metierre C, Feng YJ, Li E, Gleave M et al: Targeting amino acid transport in metastatic castration-resistant prostate cancer: effects on cell cycle, cell growth, and tumor development. Journal of the National Cancer Institute 2013, 105(19):1463-1473.

17. Kidokoro T, Tanikawa C, Furukawa Y, Katagiri T, Nakamura Y, Matsuda K: CDC20, a potential cancer therapeutic target, is negatively regulated by p53. Oncogene 2008, 27(11):1562-1571. 
18. Diboun I, Wernisch L, Orengo CA, Koltzenburg M: Microarray analysis after RNA amplification can detect pronounced differences in gene expression using limma. BMC genomics 2006, 7:252.

19. Chalmers ZR, Connelly CF, Fabrizio D, Gay L, Ali SM, Ennis R, Schrock A, Campbell B, Shlien A, Chmielecki $\mathrm{J}$ et al: Analysis of 100,000 human cancer genomes reveals the landscape of tumor mutational burden. Genome medicine 2017, 9(1):34.

20. Dudley JC, Lin MT, Le DT, Eshleman JR: Microsatellite Instability as a Biomarker for PD-1 Blockade. Clinical cancer research : an official journal of the American Association for Cancer Research 2016, 22(4):813-820.

21. Baghban R, Roshangar L, Jahanban-Esfahlan R, Seidi K, Ebrahimi-Kalan A, Jaymand M, Kolahian S, Javaheri T, Zare P: Tumor microenvironment complexity and therapeutic implications at a glance. Cell communication and signaling : CCS 2020, 18(1):59.

22. Hoeller $D$, Hecker $C M$, Dikic I: Ubiquitin and ubiquitin-like proteins in cancer pathogenesis. Nature reviews Cancer 2006, 6(10):776-788.

23. Nakayama KI, Nakayama K: Ubiquitin ligases: cell-cycle control and cancer. Nature reviews Cancer 2006, 6(5):369-381.

24. Zhang J, Wan L, Dai X, Sun Y, Wei W: Functional characterization of Anaphase Promoting Complex/Cyclosome (APC/C) E3 ubiquitin ligases in tumorigenesis. Biochimica et biophysica acta 2014, 1845(2):277-293.

25. Chang L, Barford D: Insights into the anaphase-promoting complex: a molecular machine that regulates mitosis. Current opinion in structural biology 2014, 29:1-9.

26. Wang L, Zhang J, Wan L, Zhou X, Wang Z, Wei W: Targeting Cdc20 as a novel cancer therapeutic strategy. Pharmacology \& therapeutics 2015, 151:141-151.

27. Clute P, Pines J: Temporal and spatial control of cyclin B1 destruction in metaphase. Nature cell biology 1999, 1(2):82-87.

28. Harley ME, Allan LA, Sanderson HS, Clarke PR: Phosphorylation of Mcl-1 by CDK1-cyclin B1 initiates its Cdc20-dependent destruction during mitotic arrest. The EMBO journal 2010, 29(14):2407-2420.

29. Chang DZ, Ma Y, Ji B, Liu Y, Hwu P, Abbruzzese JL, Logsdon C, Wang H: Increased CDC20 expression is associated with pancreatic ductal adenocarcinoma differentiation and progression. Journal of hematology \& oncology 2012, 5:15.

30. Karra H, Repo H, Ahonen I, Löyttyniemi E, Pitkänen R, Lintunen M, Kuopio T, Söderström M, Kronqvist $P$ : Cdc20 and securin overexpression predict short-term breast cancer survival. British journal of cancer 2014, 110(12):2905-2913.

31. Kato T, Daigo Y, Aragaki M, Ishikawa K, Sato M, Kaji M: Overexpression of CDC20 predicts poor prognosis in primary non-small cell lung cancer patients. Journal of surgical oncology 2012, 106(4):423-430.

32. Liu L, Bai X, Wang J, Tang XR, Wu DH, Du SS, Du XJ, Zhang YW, Zhu HB, Fang Y et al: Combination of TMB and CNA Stratifies Prognostic and Predictive Responses to Immunotherapy Across 
Metastatic Cancer. Clinical cancer research : an official journal of the American Association for Cancer Research 2019, 25(24):7413-7423.

33. Snyder A, Makarov V, Merghoub T, Yuan J, Zaretsky JM, Desrichard A, Walsh LA, Postow MA, Wong P, Ho TS et al: Genetic basis for clinical response to CTLA-4 blockade in melanoma. The New England journal of medicine 2014, 371(23):2189-2199.

34. Samstein RM, Lee CH, Shoushtari AN, Hellmann MD, Shen R, Janjigian YY, Barron DA, Zehir A, Jordan EJ, Omuro A et al: Tumor mutational load predicts survival after immunotherapy across multiple cancer types. Nature genetics 2019, 51(2):202-206.

35. Shen H, Zhong M, Wang W, Liao P, Yin X, Rotroff D, Knepper TC, McLeod HL, Zhou C, Xie S et al: EBV infection and MSI status significantly influence the clinical outcomes of gastric cancer patients. Clinica chimica acta; international journal of clinical chemistry 2017, 471:216-221.

36. Zhang CM, Lv JF, Gong L, Yu LY, Chen XP, Zhou HH, Fan L: Role of Deficient Mismatch Repair in the Personalized Management of Colorectal Cancer. International journal of environmental research and public health 2016, 13(9).

37. Quail DF, Joyce JA: Microenvironmental regulation of tumor progression and metastasis. Nature medicine 2013, 19(11):1423-1437.

38. Li D, Zhu J, Firozi PF, Abbruzzese JL, Evans DB, Cleary K, Friess H, Sen S: Overexpression of oncogenic STK15/BTAK/Aurora A kinase in human pancreatic cancer. Clinical cancer research : an official journal of the American Association for Cancer Research 2003, 9(3):991-997.

39. Kwan PS, Lau CC, Chiu YT, Man C, Liu J, Tang KD, Wong YC, Ling MT: Daxx regulates mitotic progression and prostate cancer predisposition. Carcinogenesis 2013, 34(4):750-759.

40. Zhang Q, Huang H, Liu A, Li J, Liu C, Sun B, Chen L, Gao Y, Xu D, Su C: Cell division cycle 20 (CDC20) drives prostate cancer progression via stabilization of $\beta$-catenin in cancer stem-like cells. EBioMedicine 2019, 42:397-407.

41. Chu Z, Zhang X, Li Q, Hu G, Lian CG, Geng S: CDC20 contributes to the development of human cutaneous squamous cell carcinoma through the Wnt/ $\beta$-catenin signaling pathway. International journal of oncology 2019, 54(5):1534-1544.

42. Sun C, Li M, Feng Y, Sun F, Zhang L, Xu Y, Lu S, Zhu J, Huang J, Wang J et al: MDM2-P53 Signaling Pathway-Mediated Upregulation of CDC20 Promotes Progression of Human Diffuse Large B-Cell Lymphoma. OncoTargets and therapy 2020, 13:10475-10487.

43. Yang G, Wang G, Xiong Y, Sun J, Li W, Tang T, Li J: CDC20 promotes the progression of hepatocellular carcinoma by regulating epithelial-mesenchymal transition. Molecular medicine reports 2021, 24(1).

44. Li J, Gao JZ, Du JL, Huang ZX, Wei LX: Increased CDC20 expression is associated with development and progression of hepatocellular carcinoma. International journal of oncology 2014, 45(4):15471555.

45. Wang L, Yang C, Chu M, Wang ZW, Xue B: Cdc20 induces the radioresistance of bladder cancer cells by targeting Fox01 degradation. Cancer letters 2021, 500:172-181. 
Figures

A

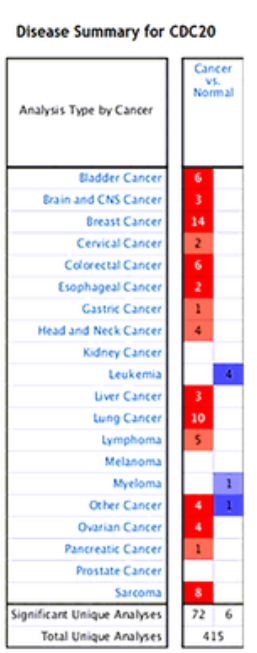

븜ำㅁำ
B

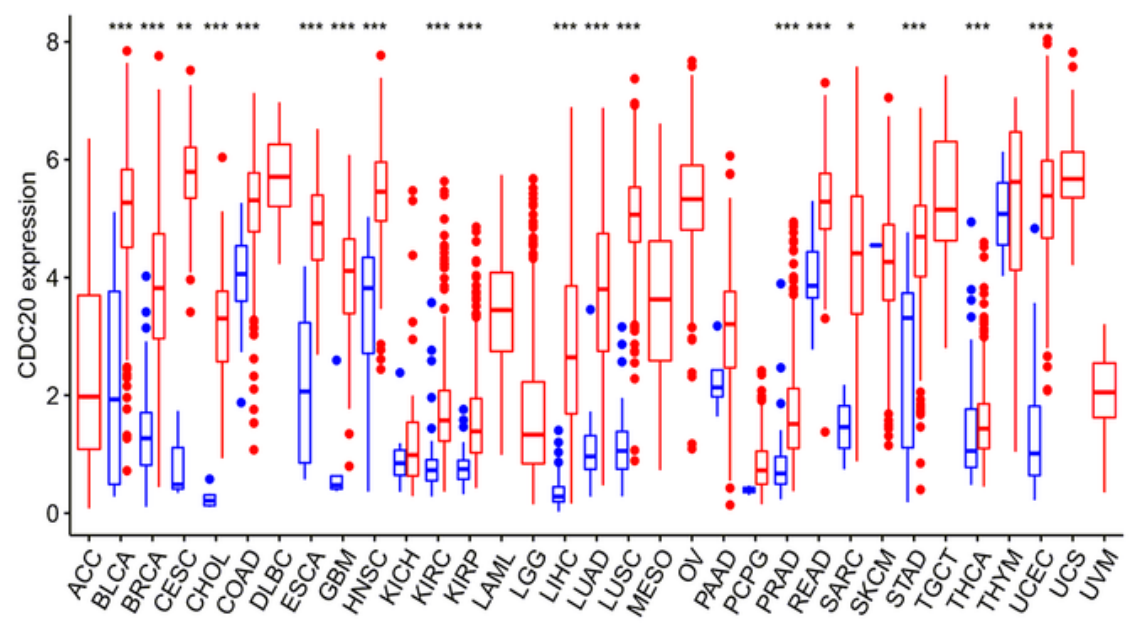

C

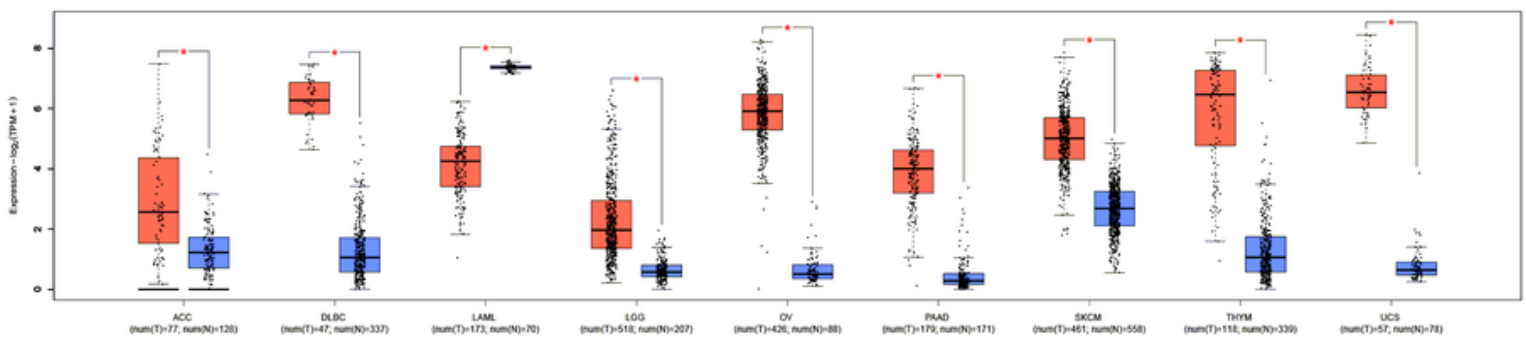

D

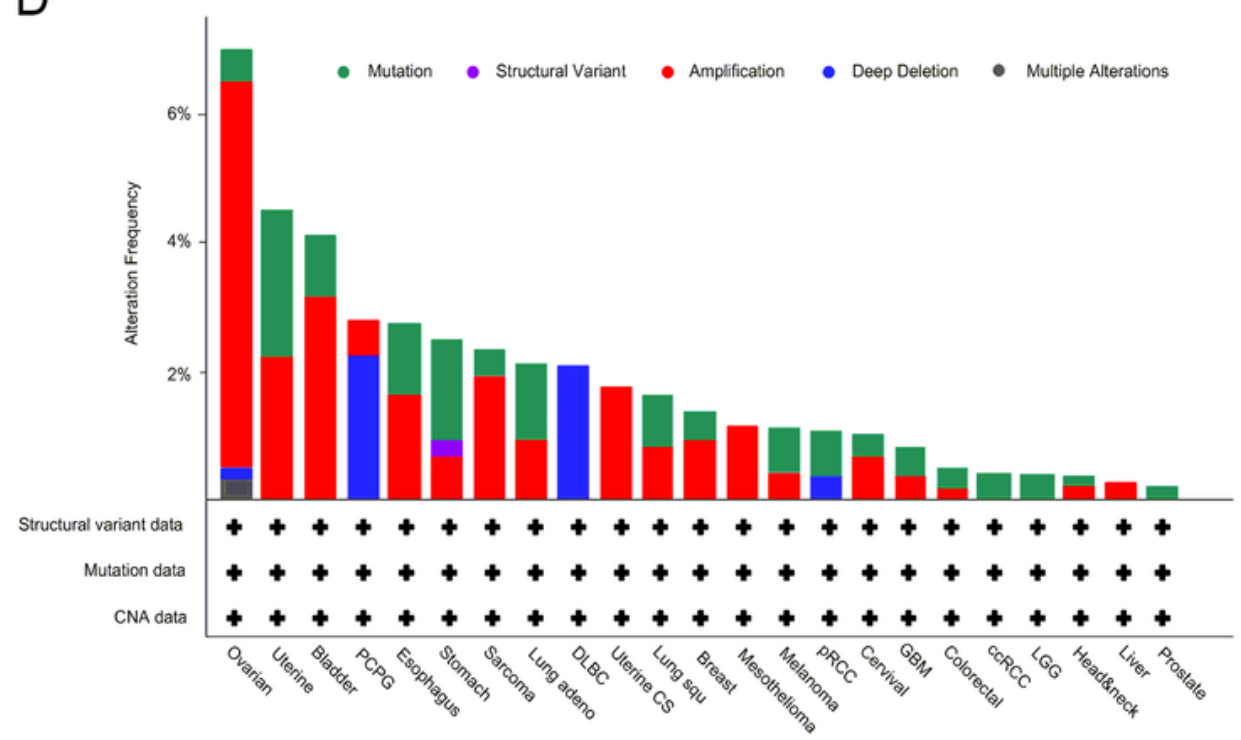

Figure 1

CDC20 expression levels and alteration in pan-cancer. The evaluation of KIF18B mRNA from Oncomine dataset (A), TCGA dataset (B) and TCGA+GTEx dataset (C). The alteration frequency of CDC20 in various cancers from the cBioPortal (D). * indicates $p<0.05$, ** indicates $p<0.01$, *** indicates $p<0.001$. 


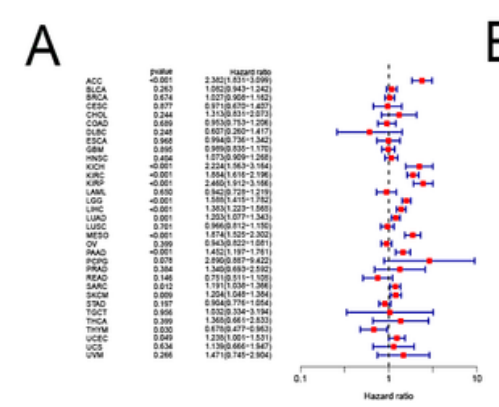

B comencec

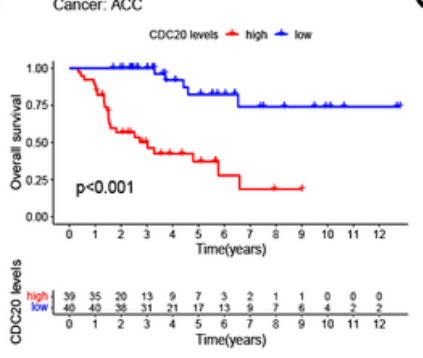

E

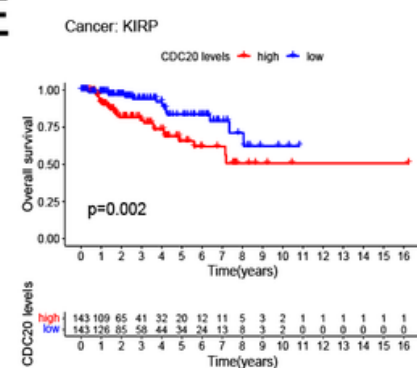

F

caneres 106

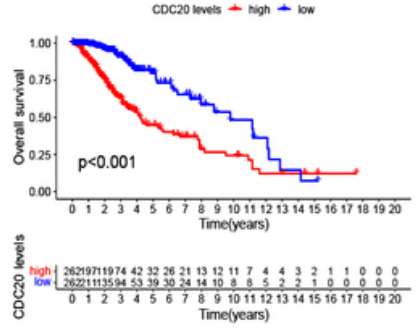

I

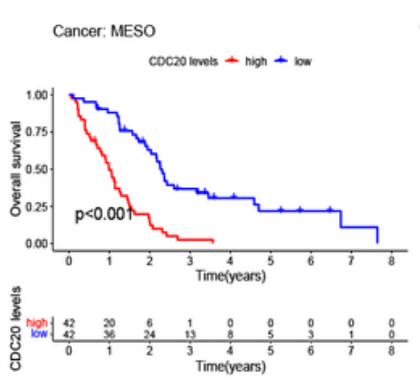

J

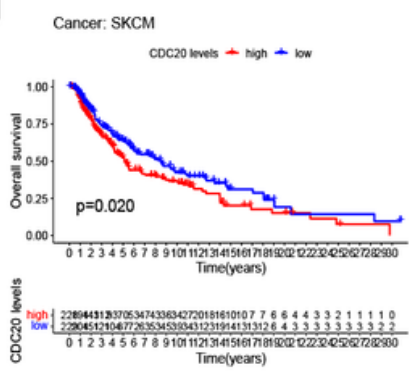

C

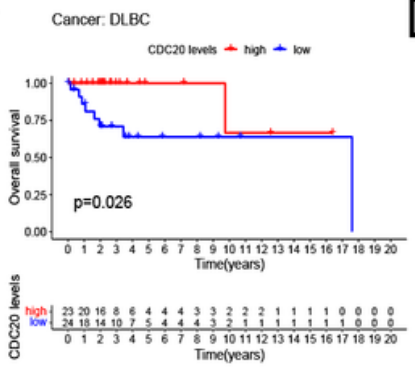

G

Cancer: UHC
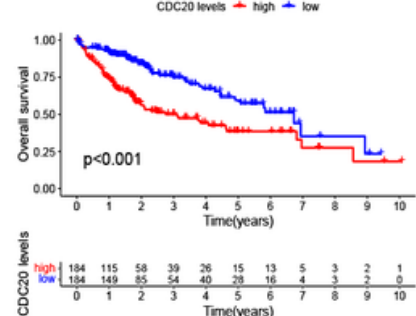

K comers rtrm

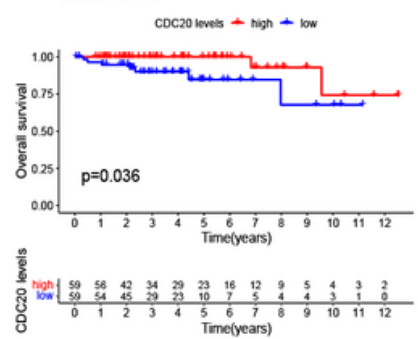

D cancer: KRPC

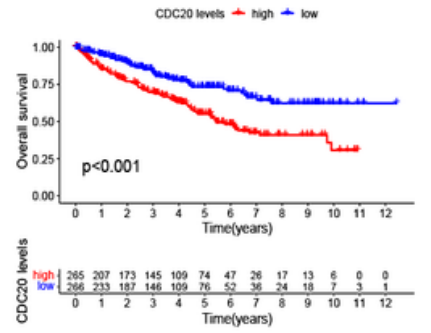

$H$ Cancer: LuAD

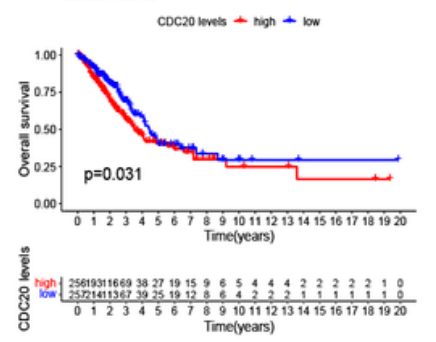

\section{Figure 2}

The correlation between CDC20 expression and overall survival time (OS) from TCGA database. (A) The forest plots of $\mathrm{CDC} 20$ in different cancers using univariate Cox regression. The survival curves of $C D C 20$ in various cancers using K-M methods. OS of ACC (B), DLBC (C), KIRC (D), KIRP (E), LGG (F), LIHC (G), LUAD (H), MESO (I), SKCM (J), THYM (K). ACC Adrenocortical carcinoma; BLCA Bladder Urothelial Carcinoma; KIRC Kidney renal clear cell carcinoma; KIRP Kidney renal papillary cell carcinoma; LGG Brain Lower Grade Glioma; LIHC Liver hepatocellular carcinoma; LUAD Lung adenocarcinoma; MESO Mesothelioma; SKCM Skin Cutaneous Melanoma; THYM Thymoma. 
A

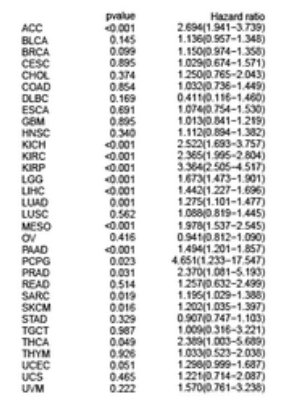

CDC20 levels + high + low
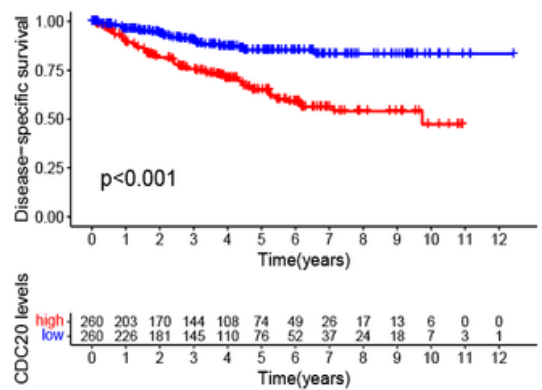

G

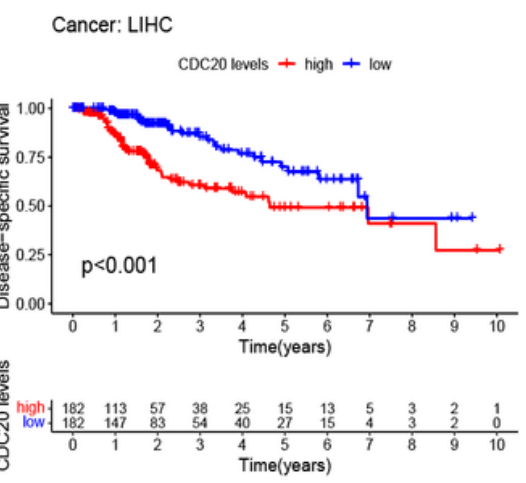

B

Cancer: ACC

CDC20 levels + high + low
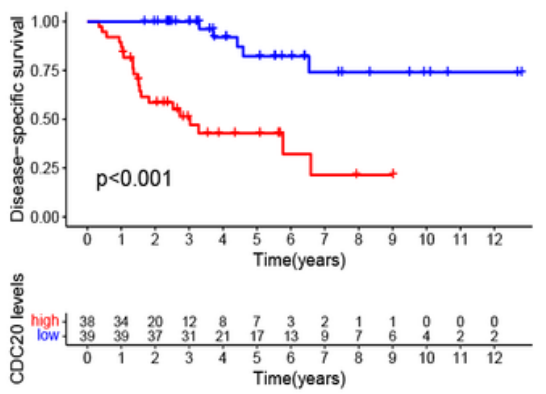

E cancer: KIRP

CDC20 levels + high + low
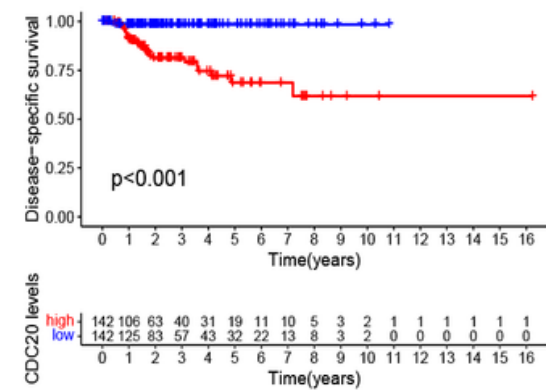

H Cancer: LUAD

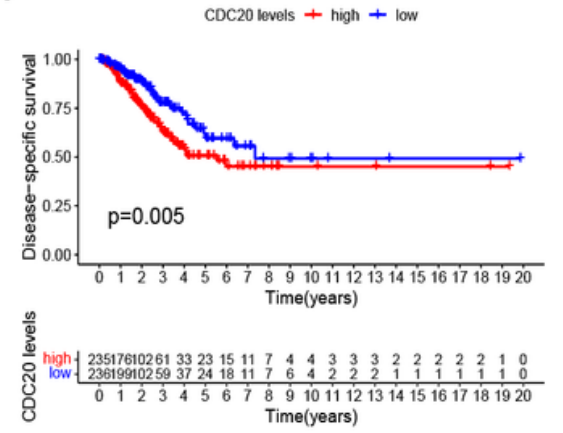

C cancer: DLBC

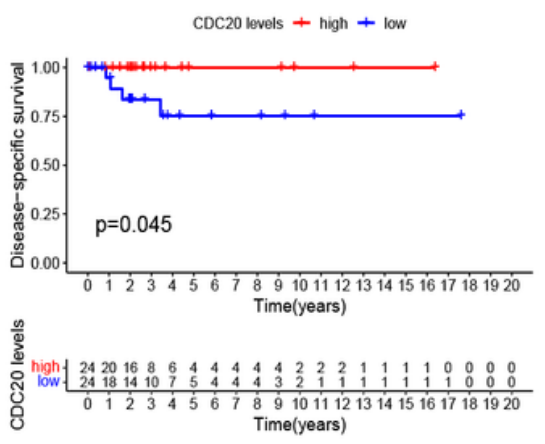

F cancer: LGG

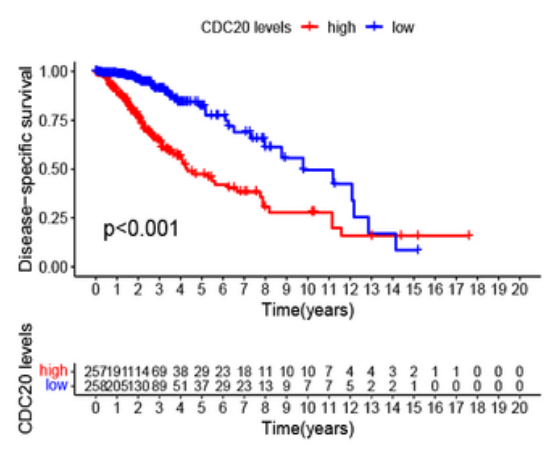

Cancer: MESO

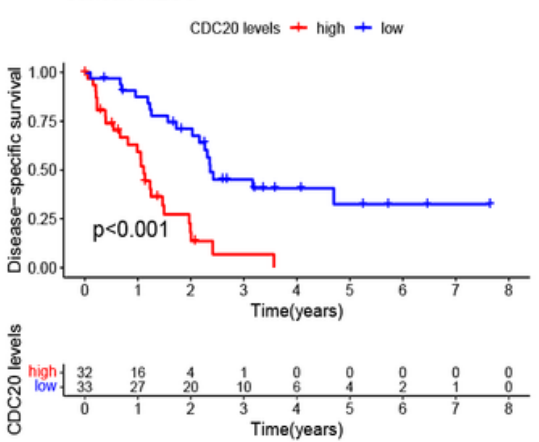

\section{Figure 3}

The correlation between CDC20 expression and Disease-Specific Survival (DSS) from TCGA database. (A) The forest plots of $\mathrm{CDC} 20$ in different cancers using univariate Cox regression. The survival curves of CDC20 in various cancers using K-M methods. DSS of ACC (B), DLBC (C), KIRC (D), KIRP (E), LGG (F), LIHC (G), LUAD (H), MESO (I). ACC Adrenocortical carcinoma; BLCA Bladder Urothelial Carcinoma; KIRC Kidney renal clear cell carcinoma; KIRP Kidney renal papillary cell carcinoma; LGG Brain Lower Grade Glioma; LIHC Liver hepatocellular carcinoma; LUAD Lung adenocarcinoma; MESO Mesothelioma. 
A

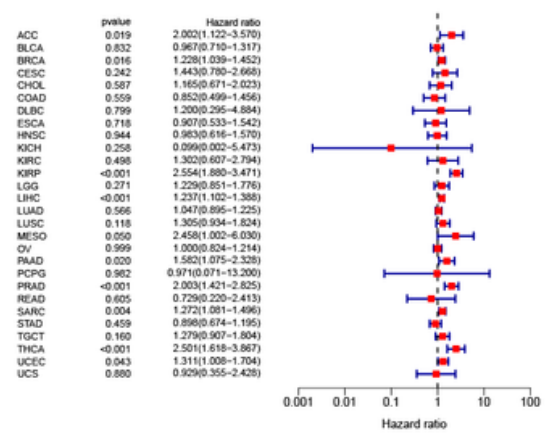

D

Cancer: LIHC
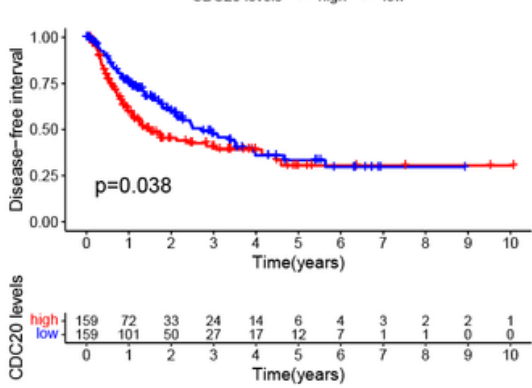

G

Cancer: SARC

CDC20 levels + high + low
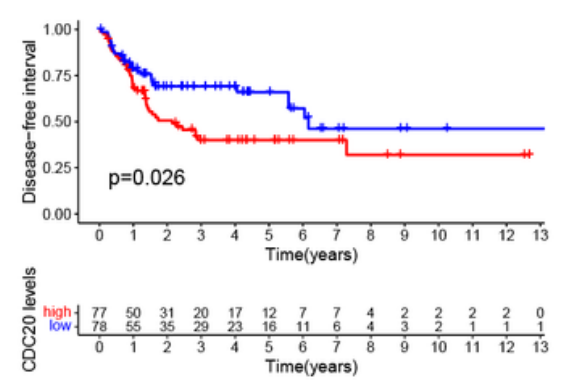

B

Cancer: ACC

CDC20 levels + high + low
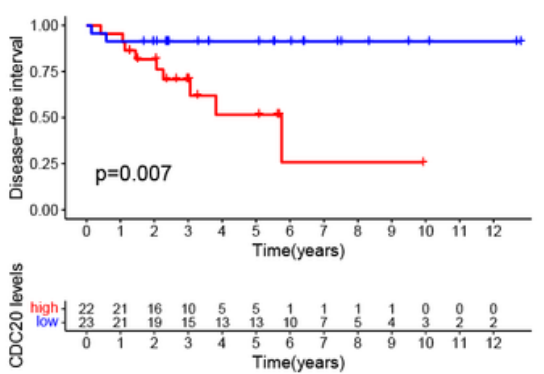

E Cancer: MESO
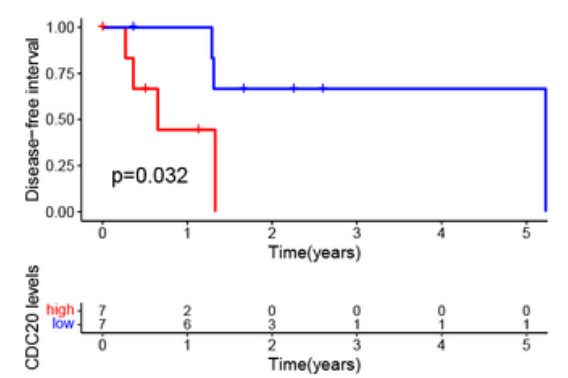

$\mathrm{H}$

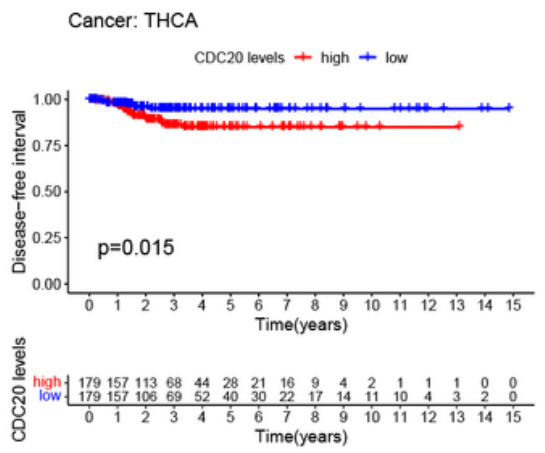

C

Cancer: KIRP

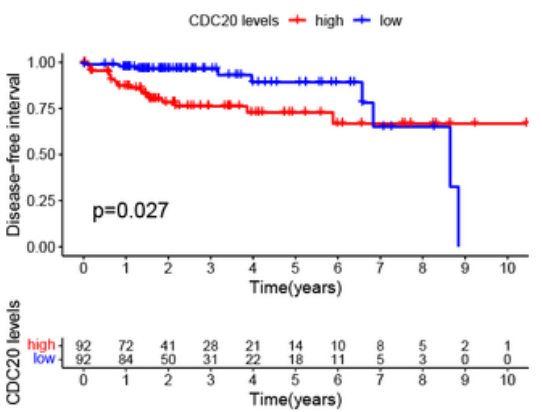

F Cancer: PRAD
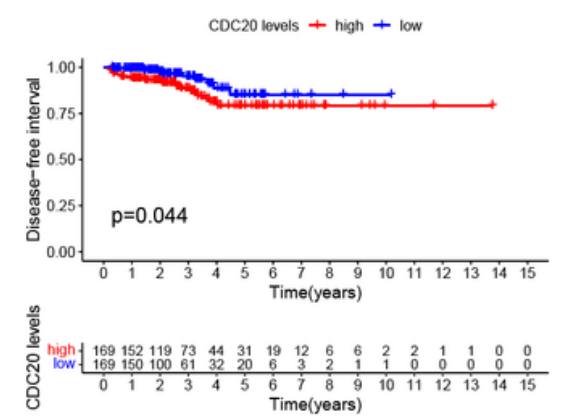

\section{Figure 4}

The correlation between CDC20 expression and Disease-Free Interval (DFI) from TCGA database. (A) The forest plots of $\mathrm{CDC} 20$ in different cancers using univariate Cox regression. The survival curves of $C D C 20$ in various cancers using K-M methods. DFI of ACC (B), KIRP (C), LIHC (D), MESO (E), PRAD (F), SARC (G), THCA (H). ACC Adrenocortical carcinoma; KIRP Kidney renal papillary cell carcinoma; LIHC Liver hepatocellular carcinoma; MESO Mesothelioma; PRAD Prostate adenocarcinoma; SARC Sarcoma; THCA Thyroid carcinoma. 


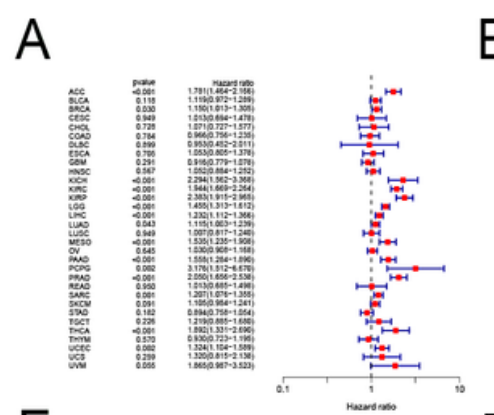

E

Canoer 160
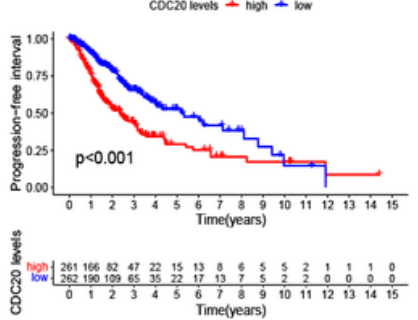

Cancer: PRAD

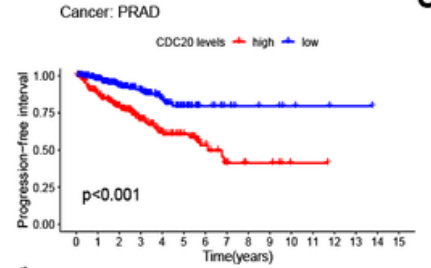

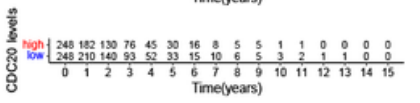

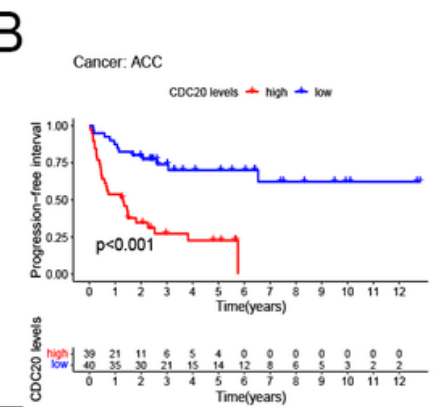

C

Cancer: KIIRC

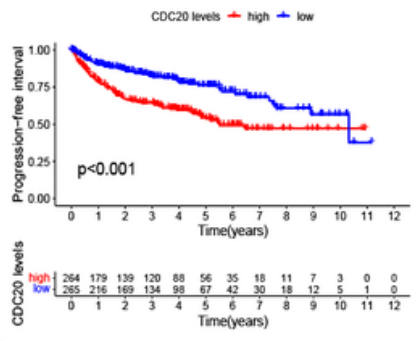

G Cancer: LHHC
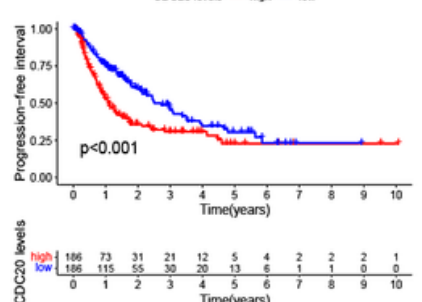

$\mathrm{J}$

Cancer: SARC
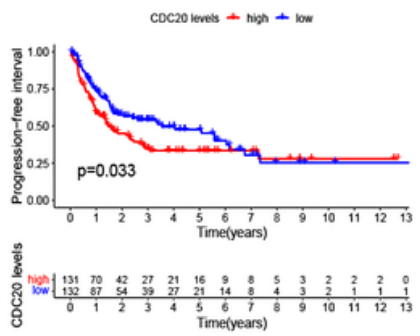

Cancer. MESO
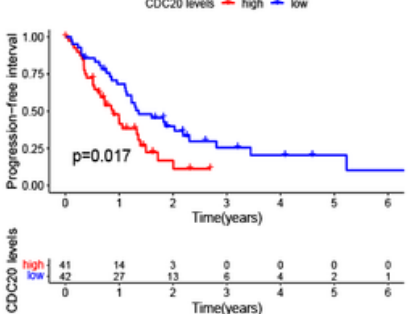

K

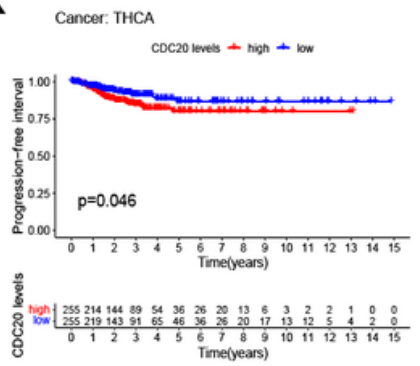

D

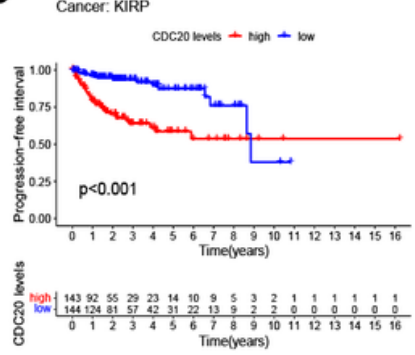

$\mathrm{H}$

Cancer: PAMD
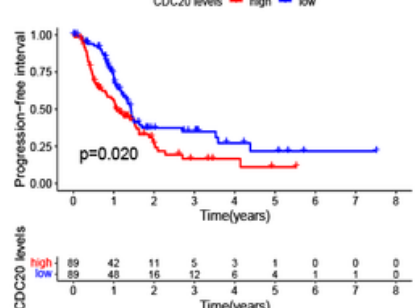

L Cancer UcEc

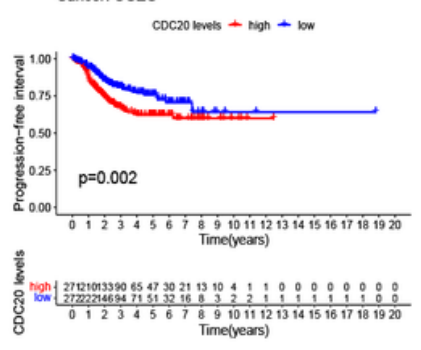

\section{Figure 5}

The correlation between CDC20 expression and Progression-Free Interval (PFI) from TCGA database. (A) The forest plots of $\mathrm{CDC20}$ in different cancers using univariate Cox regression. The survival curves of CDC20 in various cancers using K-M methods. PFI of ACC (B), KIRC (C), KIRP (D), LGG (E), LIHC (F), MESO $(G)$, PAAD $(H)$, PRAD (I), SARC (J), THCA (K), UCEC (L). ACC Adrenocortical carcinoma; KIRC Kidney renal clear cell carcinoma; KIRP Kidney renal papillary cell carcinoma; LGG Brain Lower Grade Glioma; LIHC Liver hepatocellular carcinoma; MESO Mesothelioma; PAAD Pancreatic adenocarcinoma; PRAD Prostate adenocarcinoma; SARC Sarcoma; THCA Thyroid carcinoma; UCEC Uterine Corpus Endometrial Carcinoma. 

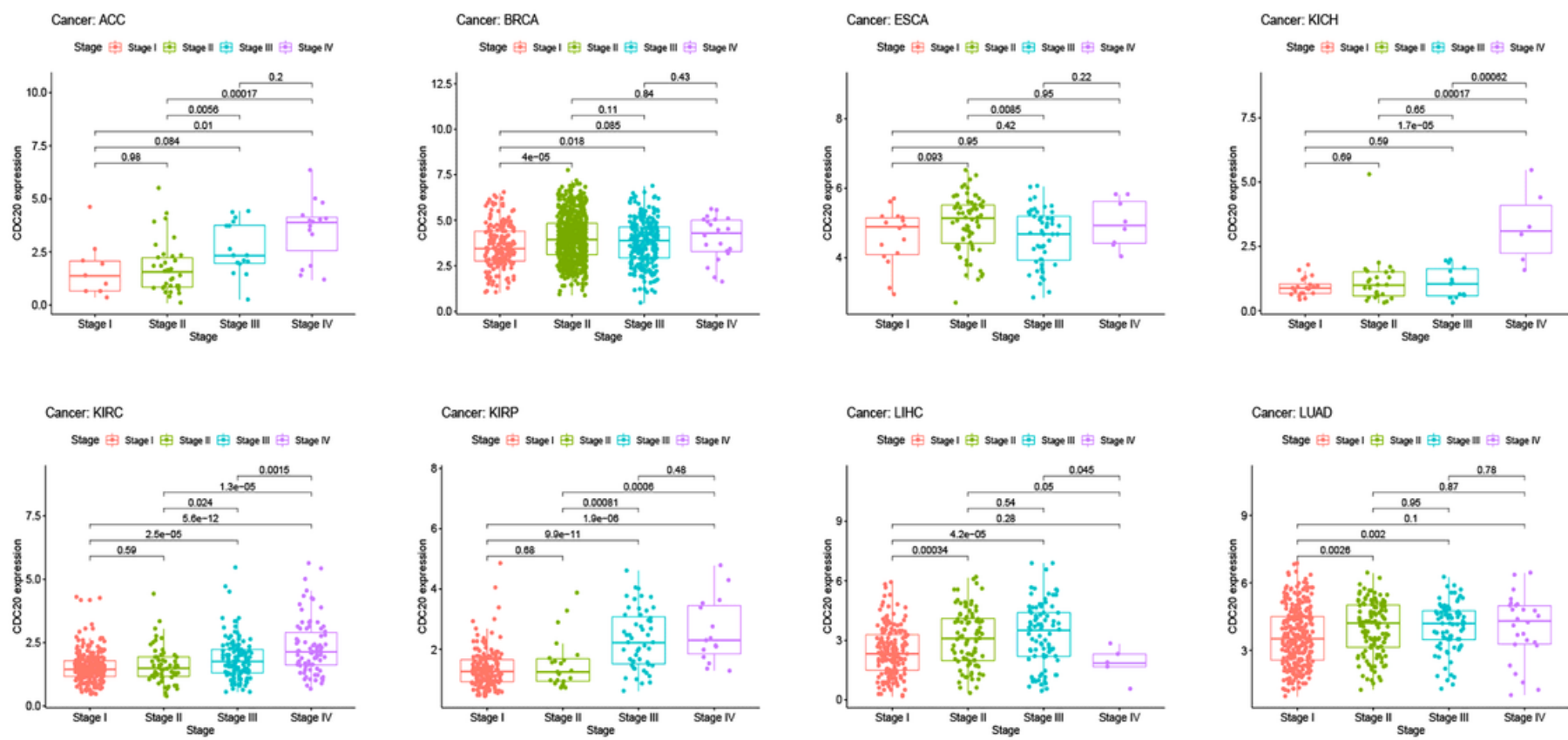

Cancer: LUAD

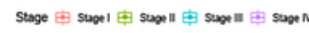
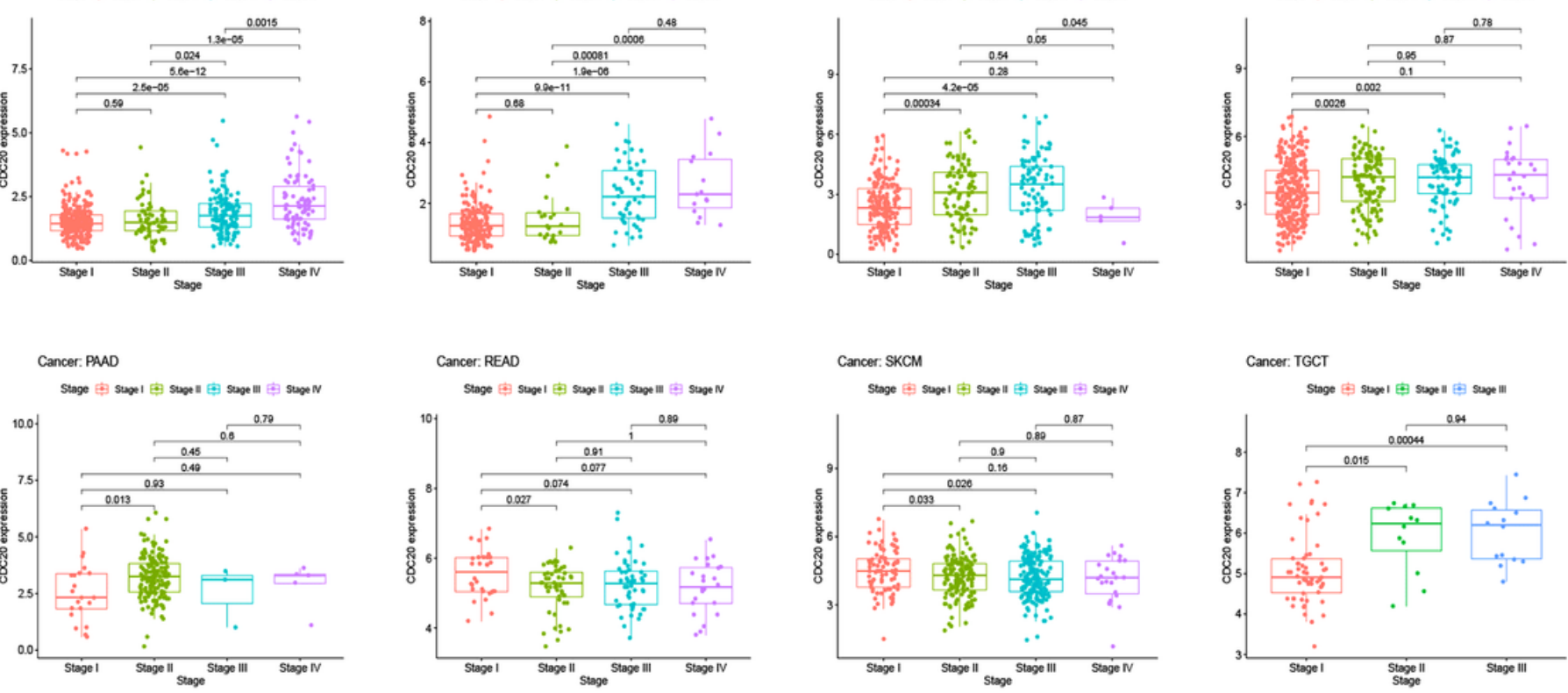

\section{Figure 6}

The correlation of CDC20 expression with clinicopathologic stage in 12 tumors. 
A

B
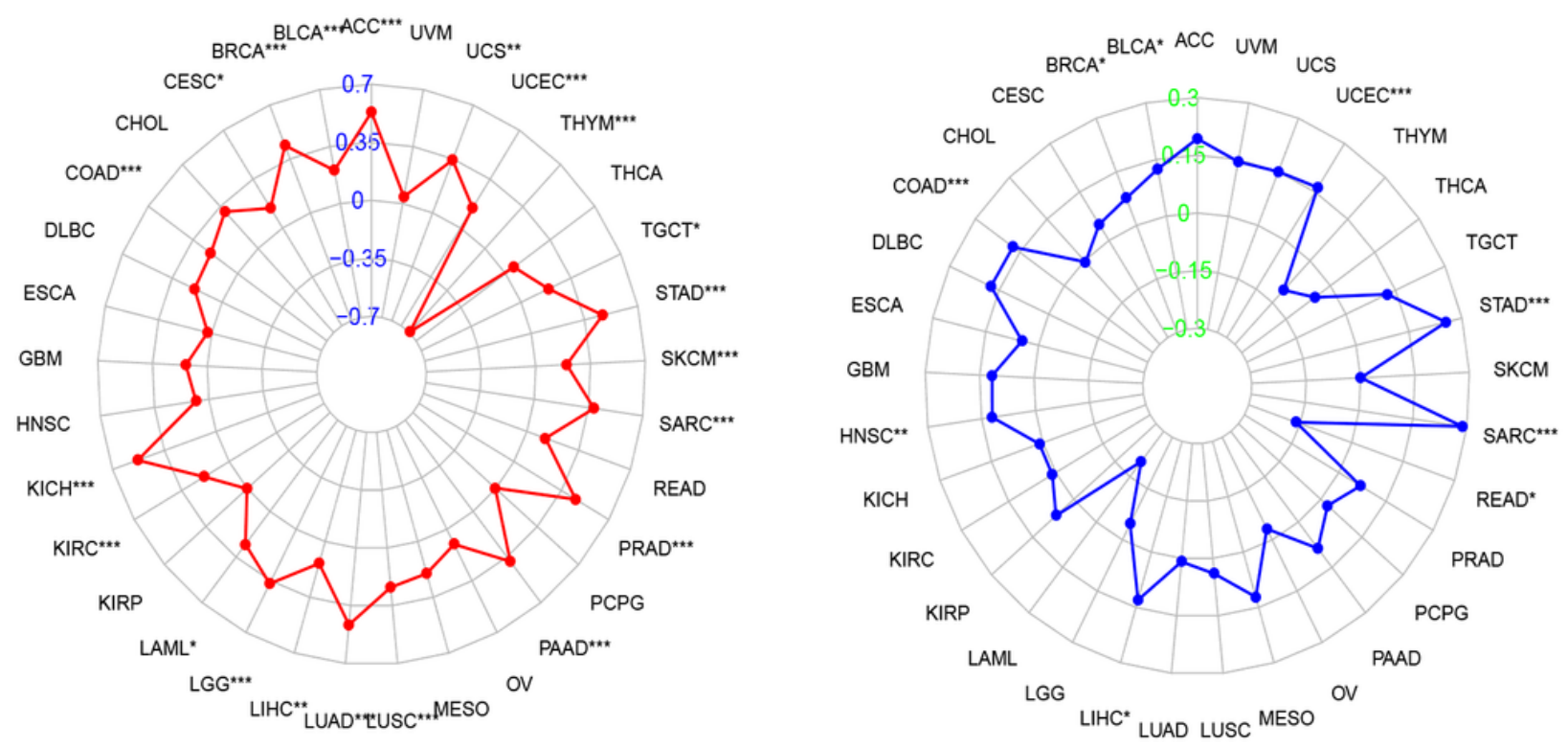

Figure 7

The relationship between $\mathrm{CDC} 20$ expression and TMB and MSI in different cancers. (A) The radar graph showed the association between TMB and CDC20 expression in various cancers. (B) The radar chart showed the association of $\mathrm{CDC} 20$ expression with MSI. The coordinate value corresponding to each point is the correlation coefficient. $\left({ }^{* \star} \mathrm{p}<0.001 ;{ }^{*} \mathrm{p}<0.01\right.$; $\left.{ }^{*} \mathrm{p}<0.05\right)$ 
Stromal score

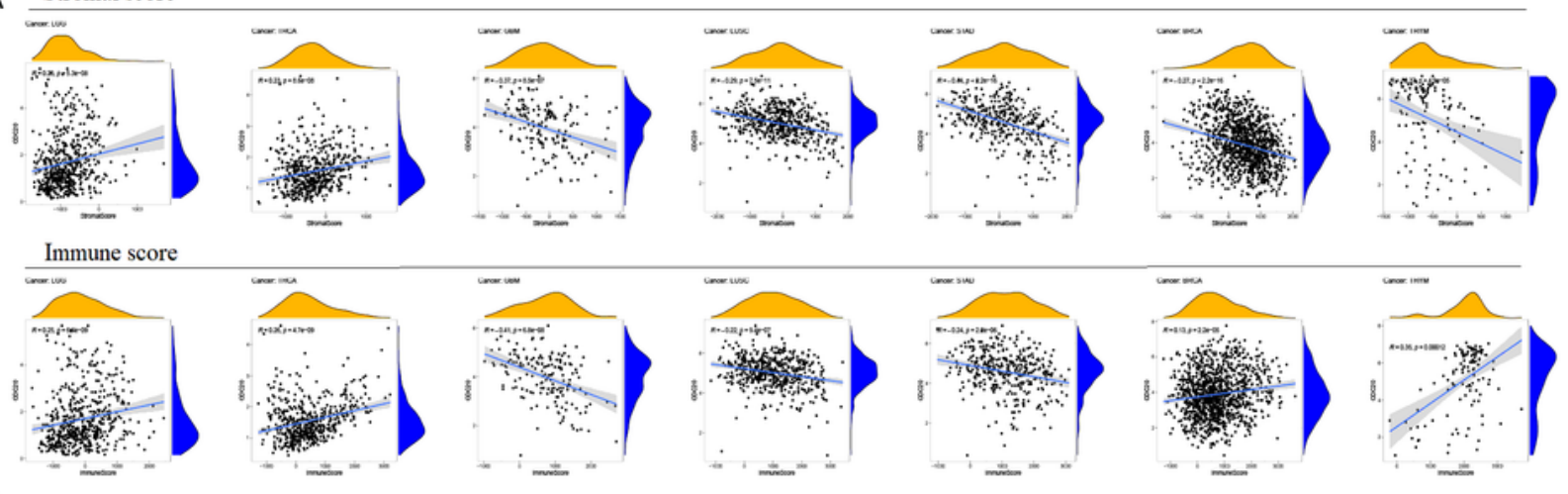

B
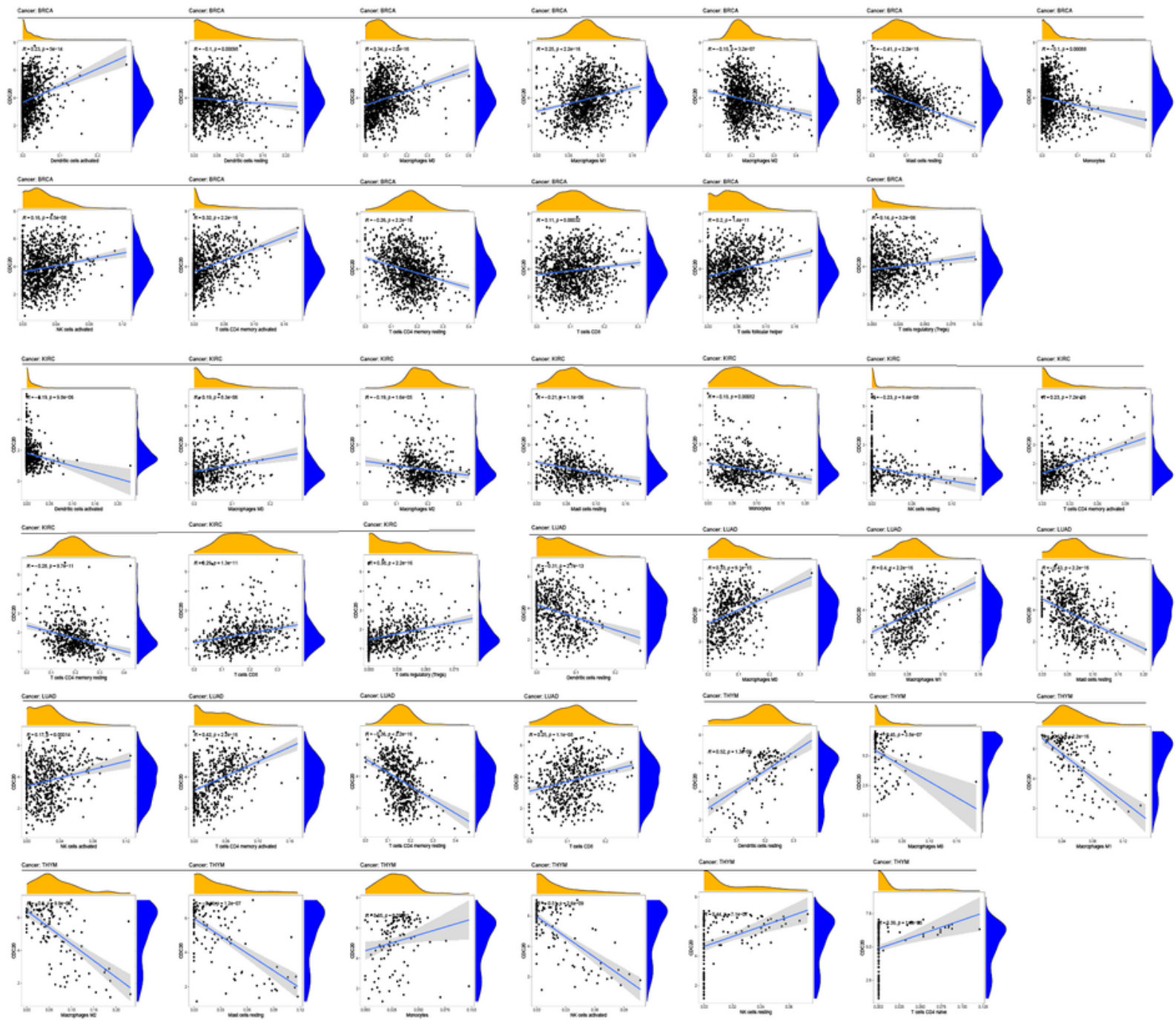

Figure 8

Correlation of CDC20 expression with tumor immune microenvironment. (A) Correlation between CDC20 expression and immune score and stromal score. (B) Correlation between CDC20 expression and infiltrating levels of immune cells in the top four tumors (BRCA, KIRC, LUAD, THYM) 
A

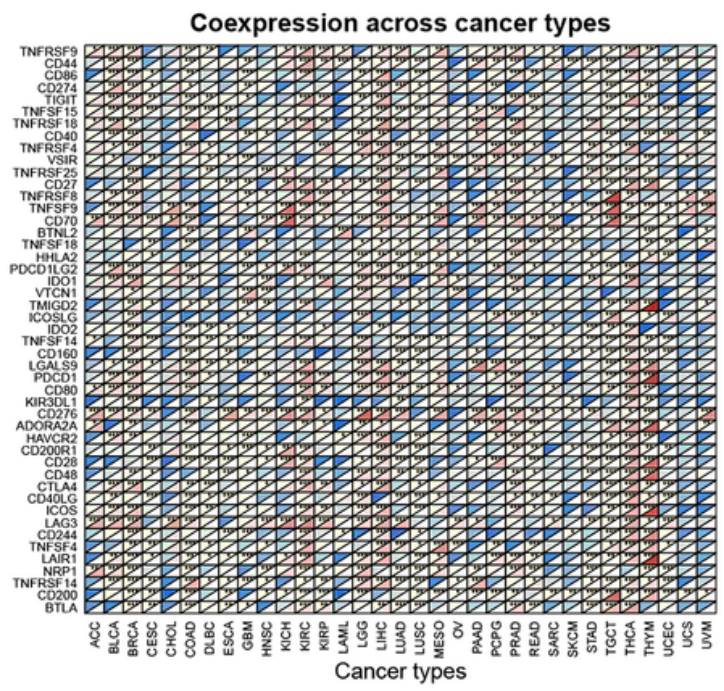

C

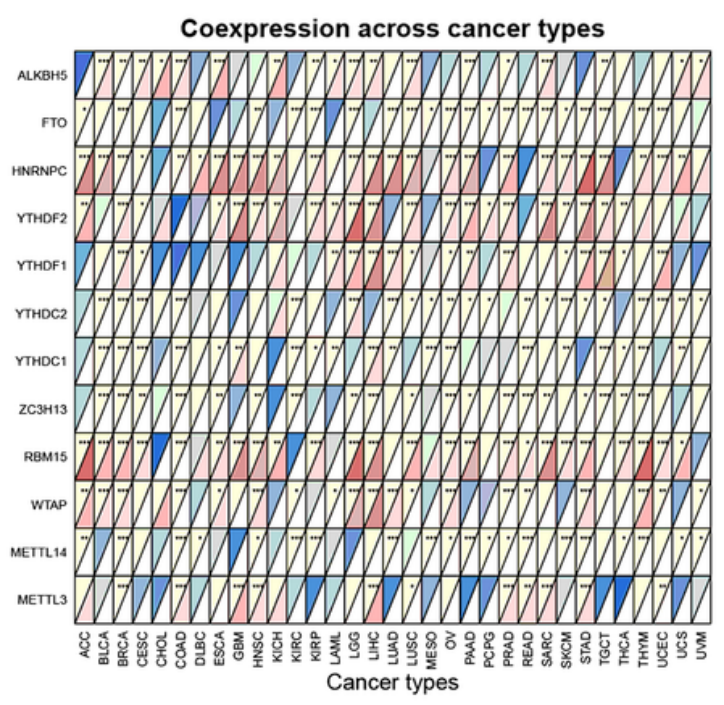

B

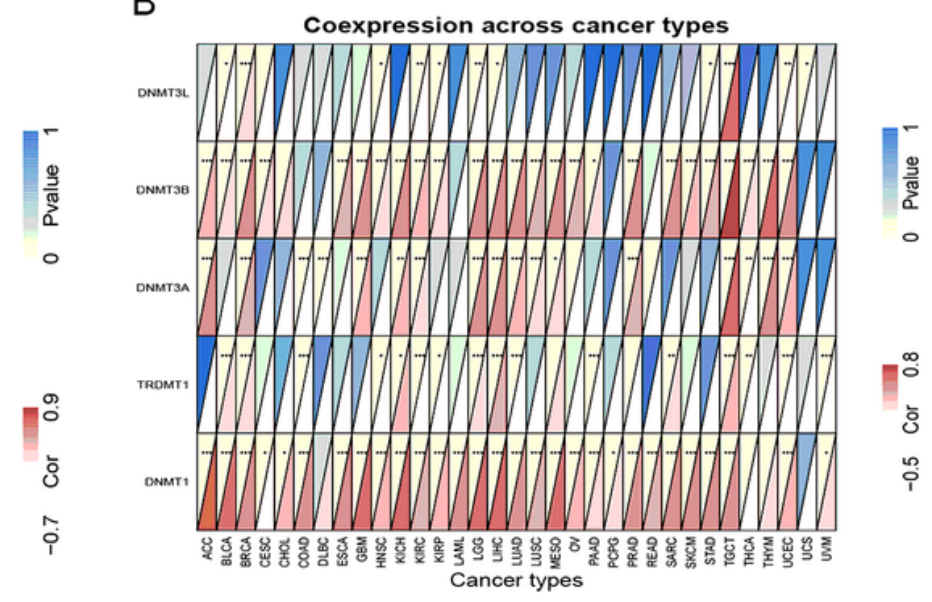

D

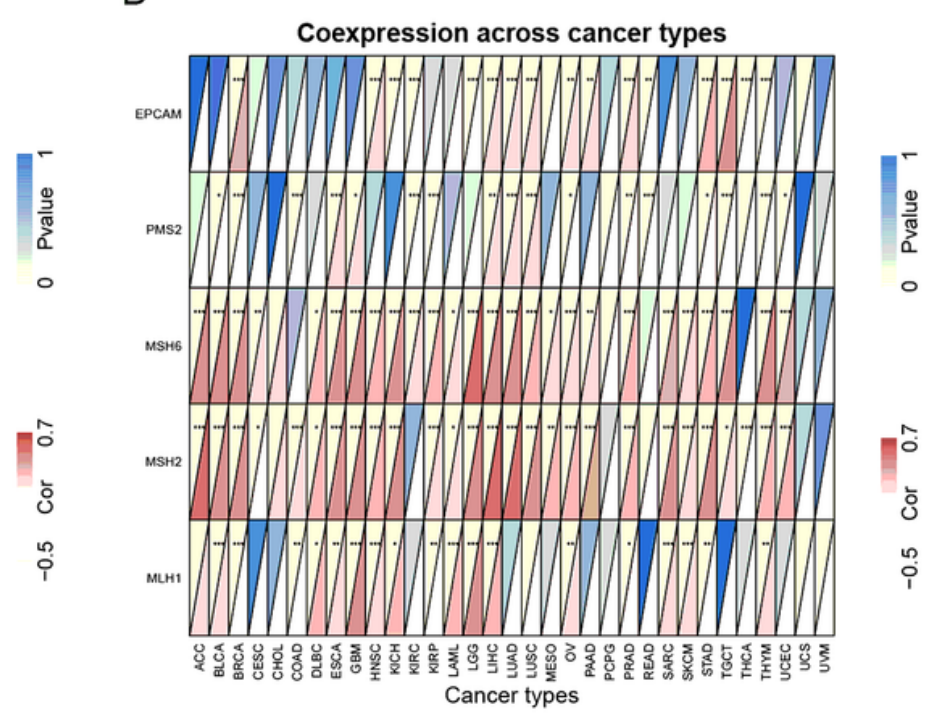

Figure 9

Co-expression of CDC20 with some specific genes in pan-cancer. (A)Co-expression of CDC20 with immune checkpoint related genes. (B) Co-expression of CDC20 with MMRs genes. (C) Co-expression of CDC20 with DNA methyltransferases. (D) Co-expression of CDC20 with mRNA m6A related genes 
$\mathrm{ACC}$

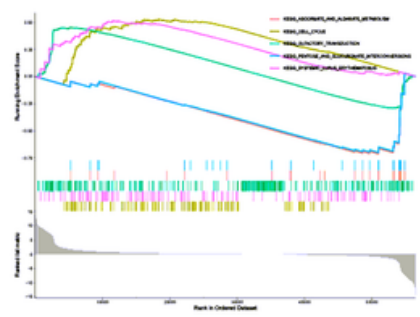

LAML

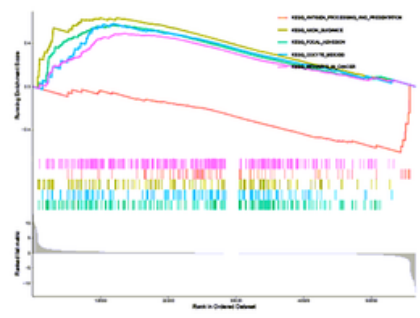

OV

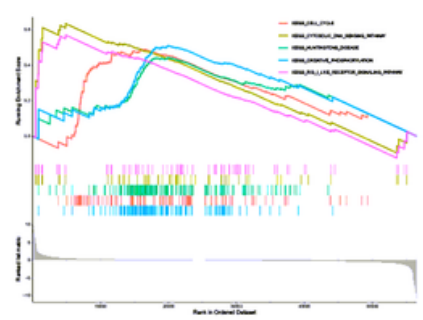

BLCA

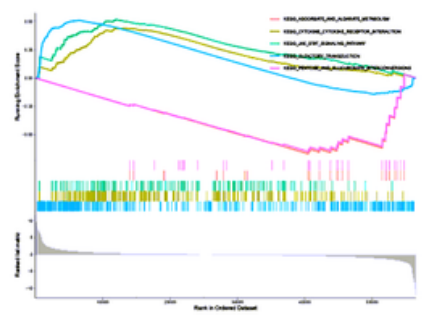

LGG

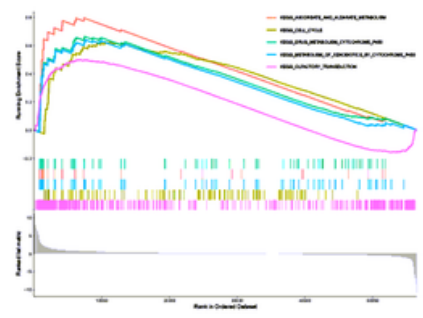

READ

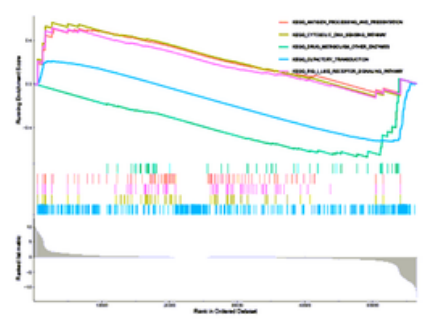

BRCA

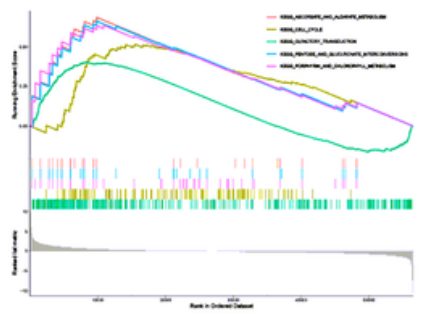

LUAD

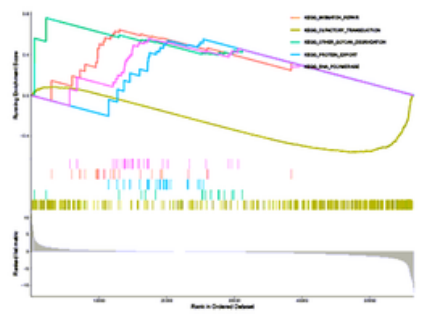

SARC

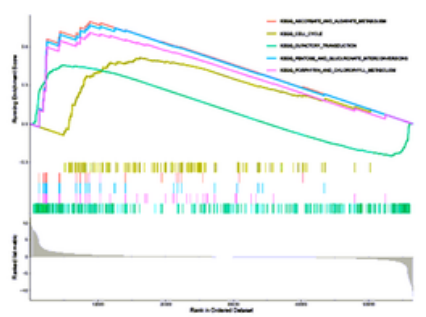

HNSC

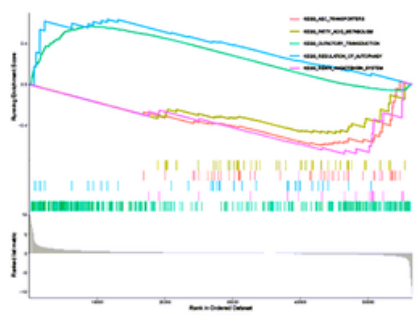

MESO

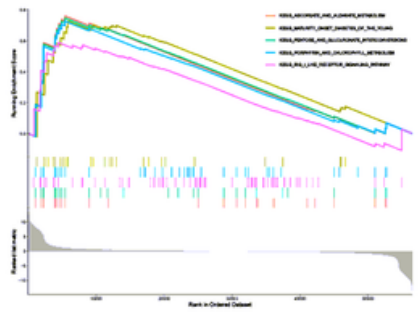

THCA

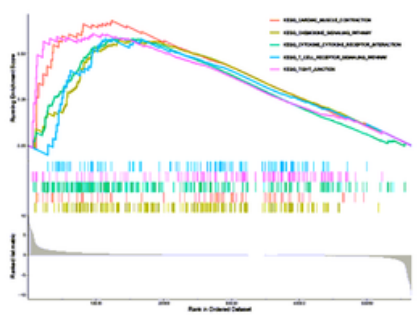

Figure 10

KEGG enrichment analysis of CDC20 in pan-cancer.

\section{Supplementary Files}

This is a list of supplementary files associated with this preprint. Click to download.

- Tables1.txt

- Tables2.txt

- Tables3.txt

- Tables4.txt

- Tables5.txt

- Tables6.txt 